\title{
SIMULACIÓN DEL FLUJO DE CALOR PARA EL REDISEÑO DE UN HORNO DE COCCIÓN DE PAN
}

\author{
Luis Cifuentes Rubio ${ }^{1}$, Alfonso Rodríguez Peña ${ }^{2}$, Lisandro Vargas Henríquez ${ }^{3}$
}

1Doctor en mecánica de fluidos. Universidad de Zaragoza-España. Icifuentes@unizar.es.

${ }^{2}$ Magister en Ingeniería Mecánica. Docente Universidad del Atlántico. Barranquilla-Colombia.

Alfonsorodriguez1@mail.uniatlantico.edu.co.

${ }^{3}$ Magister en Materiales y Procesos. Docente Universidad del Atlántico. Barranquilla-Colombia.

lisandrovargas@mail.uniatlantico.edu.co.

\section{RESUMEN}

En la industria panificadora de nuestro país, es muy común encontrar procesos de producción que se ven afectados de forma directa e indirecta por el bajo nivel de tecnificación que existe en cuanto al diseño y construcción de los hornos. Hecho que se ve reflejado en los inconvenientes que tienen lugar durante el proceso de horneo. La cocción es un proceso que juega un papel importante en la calidad del producto, el cual es influenciado principalmente por la temperatura durante la cocción por lotes. Por lo tanto, en el presente artículo se muestra la evaluación de los perfiles de temperatura y velocidad del flujo al interior del horno y se verifican los problemas que se presentan en la transferencia de calor de los panes mediante la herramienta computacional CFD (Computational Fluid Dynamics), para de esta manera proponer tres alternativas de rediseño del horno que redunden en mejoras de la distribución del flujo y los procesos de transferencia de calor y momentum en su interior, a fin de establecer un marco comparativo que permita seleccionar la propuesta más óptima para el proceso de cocción.

Palabras clave: Dinámica de fluidos computacional (CFD); uniformidad del flujo de calor; campo de flujo Recibido: 30 de Septiembre de 2018. Aceptado: 30 de Noviembre de 2018

Received: September 30, 2018. Accepted: November 30, 2018

\section{SIMULATION OF HEAT FLOW FOR THE REDESIGN OF A BREAD BAKING OVEN}

\begin{abstract}
In the bread industry of our country, it is very common to find production processes that are directly and indirectly affected by the low level of technology that exists in terms of the design and construction of the ovens. This fact is reflected in the inconveniences that take place during the baking process. Baking is a process that plays an important role in the quality of the product, which is mainly influenced by the temperature during batch cooking. Therefore, this paper shows the evaluation temperature profiles and flow velocity inside the oven and verifies the problems that arise in the heat transfer of the breads by means of the computational tool CFD (Computational Fluid Dynamics), in order to propose three alternatives for redesigning the oven that result in improvements in the distribution of the flow and the processes of heat transfer and momentum inside it, in order to establish a comparative framework that allows selecting the most optimal proposal for the baking process.
\end{abstract}

Keywords: Computational fluids dynamics (CFD); uniformity of heat flow; flow field

Cómo citar este artículo: L. Cifuentes, A. Rodriguez, L. Vargas. "Simulación del flujo de calor para el rediseño de un horno de cocción de pan", Revista Politécnica, vol. 15, no.28 pp.97-106, 2019. DOI: https://doi.org/10.33571/rpolitec.v15n28a9 


\section{INTRODUCCIÓN}

Las empresas panificadoras locales poseen un escaso nivel tecnológico que no les permite definir nuevas alternativas conducentes al mejoramiento de aspectos concernientes a su quehacer diario, por esta razón es factible encontrar procesos rudimentarios basados en el empirismo, equipos de horneo que presentan inconvenientes a nivel de diseño que se reflejan en problemas en el proceso de cocción de los panes y que por ende tienen trascendencia en la calidad del producto final. Estos hornos disponen de varias zonas de calentamiento independientes, donde el flujo de calor se divide en canales de calentamiento superior e inferior [1].

Un aspecto importante para los hornos de panadería es la búsqueda de las condiciones óptimas de funcionamiento. Los criterios principales que determinan el horno son: la uniformidad del flujo de calor en el ancho de la cámara de cocción; ajuste del flujo de calor en función de la fase de cocción de los productos; bajo consumo de combustible.

La distribución no uniforme del calor en el interior del horno provoca variaciones en el contenido de humedad y el color del pan. Estas variaciones en la calidad del pan pueden minimizarse mediante el diseño adecuado del horno, así como manteniendo las condiciones de procesamiento adecuadas, como la temperatura del aire, la potencia calorífica, el tiempo de cocción y el tamaño del pan [2-6].

Al efectuar una observación de las investigaciones existentes en torno al estudio del flujo de calor en hornos, se nota que la mayoría se perfilan hacia el empleo de herramientas CFD y hacia investigar el proceso de cocción [4-9]. CFD es una herramienta numérica que puede predecir el flujo de fluidos, la transferencia de calor y masa, las reacciones químicas y otros fenómenos mediante la resolución de un conjunto de ecuaciones matemáticas determinantes (conservación de la masa, momentum y energía) para celdas discretizadas sobre un espacio definido y un dominio del tiempo utilizando [10-11]. Sin embargo, no enfatizan sobre el diseño del horno siendo uno de los principales factores responsables de la calidad del pan.

Por lo tanto, el presente trabajo tiene como objeto simular por medio de la Dinámica de Fluidos Computacional en 3D el comportamiento del flujo interno de un horno para la cocción de pan, analizando las características del proceso de transferencia de calor y de momentum, para proponer modificaciones estructurales al diseño que conducen al desarrollo de tres alternativas de fabricación orientadas al mejoramiento de la uniformidad y distribución del flujo.

\section{MATERIALES Y METODOS}

Modelo físico: En la Fig.1 se observa como está constituido estructuralmente el horno marca Weston objeto de estudio.

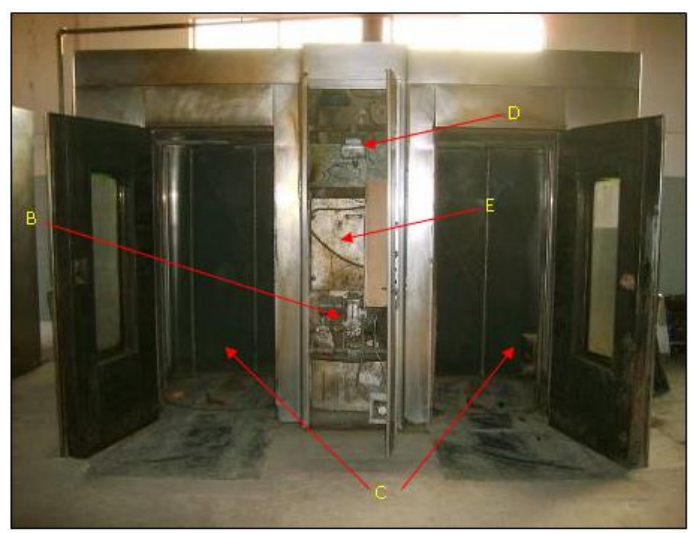

Fig. 1. Horno de fabricación de pan

El horno consta de dos cámaras (letra C), en las cuales se ubican los clavijeros con las bandejas de pan. Tiene un sistema de calentamiento a gas (letra B) que está relacionado con un indicador de temperatura (letra D). Tiene un sistema compartido de alimentación del flujo de aire caliente consistente en un intercambiador de calor de tubos (letra E) a través de los cuales circula una corriente de aire impulsada por un ventilador centrifugo. Las cámaras poseen una estructura en su interior que es la encargada de contener al clavijero y servirle de soporte. Esta consiste básicamente en un platón circular ubicado en la parte inferior del horno, conectada a un eje que va a un sistema reductor de velocidad, con el fin de brindarle al clavijero un movimiento circular de muy bajas revoluciones, alrededor de $0.063 \mathrm{rev} / \mathrm{seg}$. El horno tiene una capacidad de horneo de 14.57 a $71.65 \mathrm{~m}^{2}$ y una capacidad para bandejas que va de 48 a 236 .

Modelo computacional: La figura 2 muestra el modelo CAD del horno. Donde se toma en estudio solo una cámara del horno aprovechando la simetría de estas. No se maneja un nivel de detalle muy alto en cuanto a modelado de superficies 
externas e internas, puertas y vidrios, ya que se considera al horno como una caja rectangular de aislante.

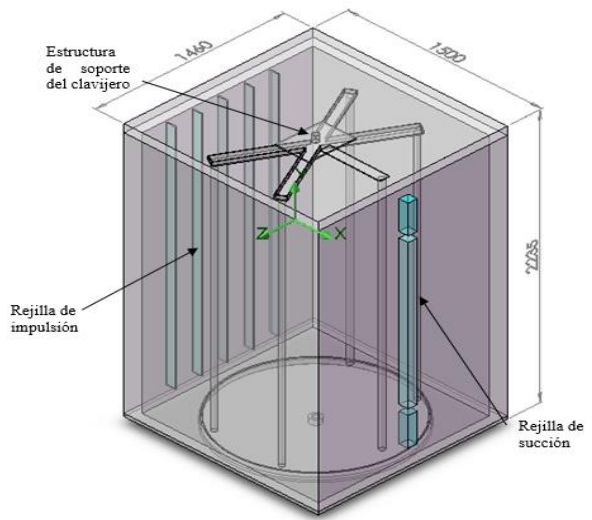

Fig. 2. Simulación CAD de una cámara del horno

Los tres modelos propuestos se analizaron en estado transitorio. Las condiciones de frontera fueron las siguientes (tabla 1).

Tabla 1. Resumen de las condiciones de simulación para las propuestas de mejora del horno

Temperatura inicial del fluido: $303^{\circ} \mathrm{K}$ $\left(30^{\circ} \mathrm{C}\right)$; Temperatura inicial del sólido: $303^{\circ} \mathrm{K} \quad\left(30^{\circ} \mathrm{C}\right)$; Velocidad inicial del aire: $0 \mathrm{~m} / \mathrm{seg}$.

Condiciones En las rejillas de succión: para la Simulación Temperatura del fluido: $303^{\circ} \mathrm{K}$ (30ํㅡ); Presión: $101325 \mathrm{~Pa}$

En las rejillas de impulsión: Temperatura del fluido: $573^{\circ} \mathrm{K}$ $\left(300^{\circ} \mathrm{C}\right)$

Todos los modelos tienen definida paredes adiabáticas y rugosidad cero.

Alternativa 1

Se aumentar el tamaño del área de succión mediante la colocación de 5 rejillas de $3 \mathrm{~cm}$ de ancho. Caudal: $0.11216 \mathrm{~m}^{3} / \mathrm{seg}$.

Alternativa 2

Se introducen paredes laterales curvas para reducir el tamaño de las paredes de succión e impulsión. Caudal: $0.11216 \mathrm{~m}^{3} / \mathrm{seg}$

Alternativa 3 Se introducen paredes laterales curvas, 10 rejillas de impulsión y 5 rejillas de succión de $10 \mathrm{~cm}$ de ancho. Caudal: $0.06231 \mathrm{~m}^{3} / \mathrm{seg}$.
Modelo matemático: Este estudio se realizó utilizando el software comercial Solidworks Flow Simulation el cual fue desarrollado para resolver las ecuaciones que rigen el comportamiento de los fluidos tanto laminar como turbulento [12-13]. Esta investigación trata precisamente de un caso turbulento y por tanto, las ecuaciones del modelo de energía y la tasa de disipación de la energía turbulenta tienen justificación para ser utilizadas en la simulación.

Las leyes de conservación de masa, momentum y energía en un sistema de coordenadas girando a una velocidad angular, $\Omega$, cerca de un eje que pasa a través del origen del sistema coordenado, pueden ser descritas según las siguientes ecuaciones [13, 14]:

$\frac{\partial \rho}{\partial t}+\frac{\partial}{\partial x_{k}}\left(\rho u_{k}\right)=0$

$\frac{\partial \rho u_{i}}{\partial t}+\frac{\partial}{\partial x_{k}}\left(\rho u_{i} u_{k}-\tau_{i k}\right)+\frac{\partial p}{\partial x_{i}}=S_{i}$

$\frac{\partial(\rho E)}{\partial t}+\frac{\partial}{\partial x_{k}}\left((\rho \mathrm{E}+\mathrm{P}) \mu_{\mathrm{k}}+q_{i}-\tau_{i k} \mu_{i}\right)=S_{k} u_{k} Q_{H}$,

Donde $\mu$ es la velocidad del fluido, $\rho$ es la densidad del fluido, $S_{i}$ es una fuerza externa distribuida por unidad de masa debido a la resistencia del medio poroso (Si $\mathrm{i}_{\text {poroso}}$ ), a una fuerza de boyamiento (Sigravedad), y al sistema de coordenadas de rotación (Sirotación); por lo tanto, $\mathrm{Si}_{\mathrm{i}}=\mathrm{Si}_{\text {poroso }}+$ Sigravedad + Sirotación. E es la energía total por unidad de masa, $Q_{H}$ es la fuente de calor por unidad de volumen, $\tau_{j k}$ es el tensor del esfuerzo cortante viscoso, qi es el flujo de calor de difusión, y los subíndices son una expresión para denotar sumatoria por encima de las tres direcciones coordenadas [13].

Dos ecuaciones de transporte adicionales son usadas para describir la energía cinética turbulenta y disipación (Ec. $(4,5))[13]$ :

$$
\begin{aligned}
& \frac{\partial \rho \kappa}{\partial t}+\frac{\partial}{\partial x_{i}}\left(\rho u_{i} \kappa\right)=\frac{\partial}{\partial x_{i}}\left(\left(\mu+\frac{\mu_{t}}{\sigma_{k}}\right) \frac{\partial \kappa}{\partial x_{i}}\right)+S_{k} \\
& \frac{\partial \rho \varepsilon}{\partial t}+\frac{\partial}{\partial x_{i}}\left(\rho u_{i} \varepsilon\right)=\frac{\partial}{\partial x_{i}}\left(\left(\mu+\frac{\mu_{t}}{\sigma_{\varepsilon}}\right) \frac{\partial \varepsilon}{\partial x_{i}}\right)+S_{\varepsilon}
\end{aligned}
$$

Donde $S_{k}$ y $S_{\varepsilon}$ son los términos de fuente. 


\section{RESULTADOS Y DISCUSIONES}

Comportamientos predominantes en el horno actual: se realizaron análisis de convergencia tanto para la selección del mallado como para la selección del paso de tiempo. Para la selección del tamaño de malla que sea adecuado para el estudio del flujo al interior del horno se llevó a cabo un proceso de refinamiento de la malla mediante la realización de 10 corridas (tabla 2). En el modelo estructural del horno se definió la ubicación de un punto de referencia con el objeto de verificar que tan constante era el valor de temperatura que se registraba en dicha posición geométrica. Para las corridas se fijaron los siguientes parámetros: temperatura del aire de entrada (Rejillas de Impulsión): $127^{\circ} \mathrm{C}\left(400{ }^{\circ} \mathrm{K}\right)$, temperatura inicial (ambiente): $30^{\circ} \mathrm{C}(303 \mathrm{~K})$ y estado estable.

Tala 2. Registro de refinamiento de malla

\begin{tabular}{ccc}
\hline $\begin{array}{c}\text { Número de } \\
\text { Corrida }\end{array}$ & $\begin{array}{c}\text { Número de } \\
\text { Celdas }\end{array}$ & $\begin{array}{c}\text { Temperatura } \\
\left({ }^{\circ} \mathrm{C}\right)\end{array}$ \\
\hline 1 & 96.617 & 399,269 \\
2 & 113.410 & 397,314 \\
3 & 121.026 & 398,972 \\
4 & 154.853 & 399,529 \\
5 & 189.983 & 399,417 \\
6 & 236.892 & 399,261 \\
7 & 238.103 & 399,24 \\
8 & 250.327 & 399,589 \\
9 & 270.175 & 399,327 \\
10 & 282.455 & 399,703 \\
\hline
\end{tabular}

Para determinar cuál de estos mallados se va utilizar, se determinó su media aritmética y ésta se compara con cada uno de los valores con el fin de establecer cual se encuentra más próximo a la media. Se seleccionó un mallado con 189.983 celdas, ya que este se encuentra un $99.99 \%$ más cerca del valor medio.

La selección del paso de tiempo adecuado se efectúa para el mallado seleccionado y los valores de paso de tiempo de: $0.1 \mathrm{seg}, 0.5 \mathrm{seg}, 0.7 \mathrm{seg}$ y 1 seg. Se establecen como referencia una ubicación en la geometría del modelo, de los cuales se extrae el valor de las variables temperatura y velocidad, para efectuar posteriormente una comparación en cada uno de los pasos de tiempo. Se encontró que no existe una variación muy notable en la variación de la temperatura para las ubicaciones seleccionadas, por lo que se puede afirmar que estos pasos de tiempo no ejercen una influencia considerable sobre los resultados obtenidos.

Para el modelo de simulación del horno, con panes en su interior, se empleó un intervalo de tiempo para el análisis de $3000 \mathrm{seg}$, que corresponde al tiempo de estabilización de calentamiento del horno más el tiempo que emplea el pan para finalizar su proceso de cocción.

Al simular las condiciones reales se identificaron fenómenos en el flujo al interior del horno, una distribución no uniforme de la velocidad y la temperatura, fig. 3.

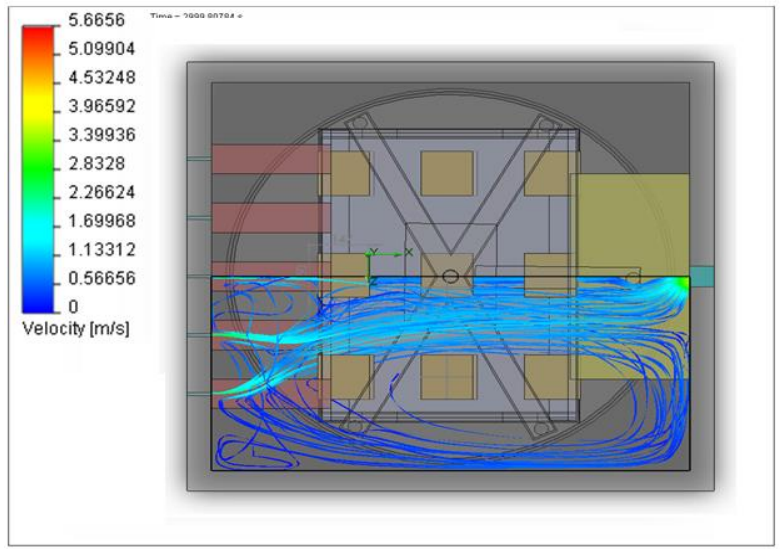

Fig. 3. Vista superior del campo de velocidad del horno actual

El aire que ingresa al horno se encuentra con fluido que inicialmente está en reposo y lo obliga a salir por las rejillas de succión induciendo así la formación de vórtices [15]; gran parte del aire no logra salir por esta rejilla cambiando de dirección, regresándose y formando una zona de recirculación de aproximadamente $43 \%$ del volumen del horno.

Este fenómeno afecta el calor aportado hacia los panes, causando un desvió de la ventilación de impulsión que se encuentran en los extremos hacia el centro del horno. Por lo tanto, los panes ubicados en esta zona, al estar expuestos a un cambio continuo del aire presentan una transferencia de calor mucho mayor que los del extremo, los cuales se encuentran en la zona de recirculación. Este hecho revela la existencia de una desigualdad en el campo de temperatura, (Fig. 4). 




Fig. 4. Campo de temperatura para el horno actual

Al evidenciarse este problema en el horno actual se propusieron cambios en su estructura los cuales causaron un efecto contundente en los patrones del flujo al interior de este.

Modelos alternativos del horno: Después del estudio del comportamiento del flujo de calor al interior del modelo que representa las características reales del horno, se proponen tres alternativas en las cuales se someten a evaluación ciertas modificaciones, cuyo objeto es contribuir a mejorar la uniformidad del flujo interno del aire.

Alternativa 1: Mayor número de rejillas de succión. Ver Fig. 5.

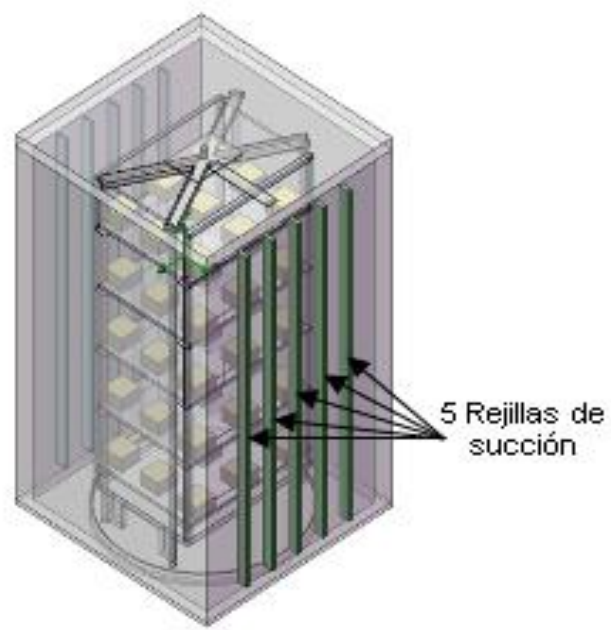

Fig. 5. Características de la alternativa 1

Este modelo se caracteriza por presentar un sistema de 5 rejillas de impulsión de aire al interior del horno, que posteriormente es succionado por un número igual de rejillas pero de menor anchura que las rejillas de este tipo en el modelo original. Esto con el fin de disminuir la resistencia al flujo que ocasiona que éste tienda a regresar hacia la impulsión. Como consecuencia de esto el flujo que se dirige hacia la succión adquiere una mayor velocidad en la zona central (ver óvalo rojo en la Fig. 6), facilitando la salida del flujo proporcionando una distribución más uniforme. Estos resultados se observan en los campos de velocidad y temperatura en las Fig. 6 y 7.

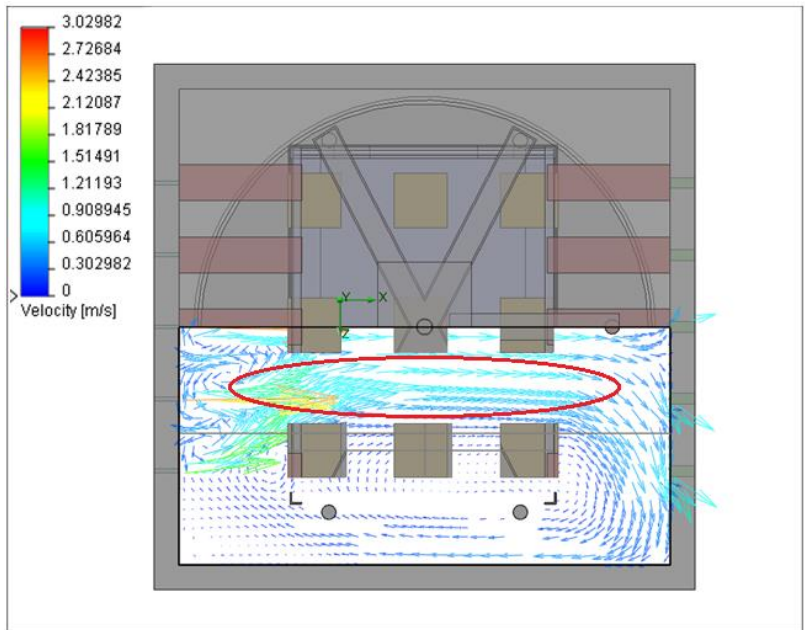

Fig. 6. Campo de velocidad para la alternativa 1

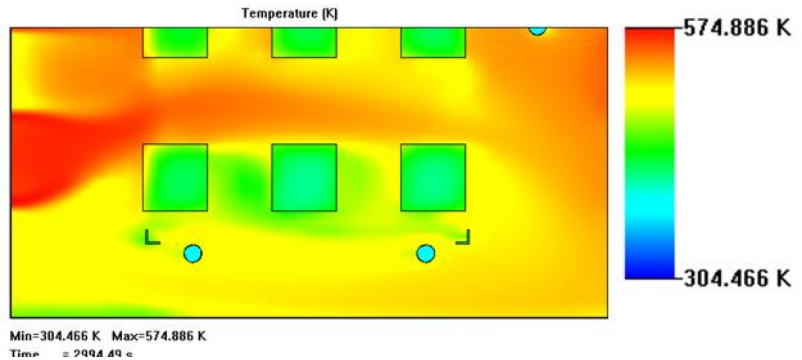

Fig. 7. Campo de temperatura alternativa 1

La Fig. 7 muestra que existe una estratificación de la temperatura en el interior del horno, donde la zona central recibe en mayor proporción el flujo caliente proveniente de la impulsión. La temperatura en la zona de recirculación es más baja que en las demás zonas; las partículas que hacen parte del flujo de la región central y la de recirculación están participando del proceso de transferencia de calor del medio hacia los panes, pero en la de recirculación las partículas no alcanzan el nivel térmico de las demás, debido a que en ésta zona es predominante la presencia de vórtices.

Alternativa 2: Horno con curvas laterales. Esta alternativa toma de la anterior el aumento del área de succión y le añade una modificación en la estructura geométrica del horno, introduciéndole 
curvas al modelo en las paredes laterales, como se muestra en la Fig. 8.

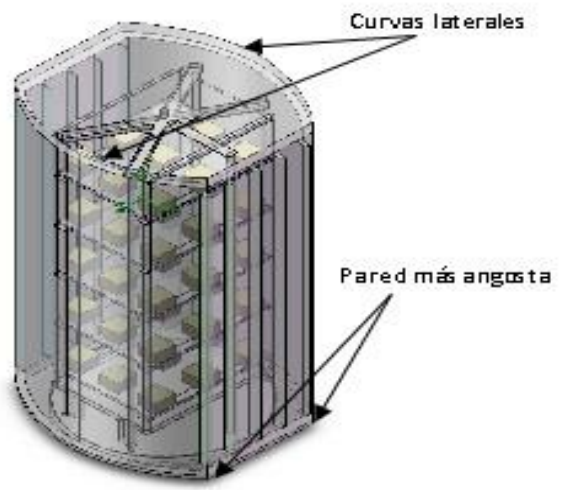

Fig. 8. Características de la alternativa 2

En esta alternativa, se toma la misma proporción y distribución de las rejillas que posee la alternativa 1 , pero la introducción de curvas en las paredes laterales, da lugar a reducir el tamaño tanto de las rejillas de succión como las de impulsión, así como el espacio ocupado por el horno en comparación con el tamaño del horno actual.

Esta modificación tiene fundamento en el problema encontrado en el horno real con respecto al flujo que se concentra en las esquinas, el cual contribuye a la formación de vórtices que consumen energía del flujo proveniente de las rejillas de impulsión, constituyéndose en entes disipadores de energía.

La introducción de paredes laterales curvas propicia un recorrido del aire con un cambio de forma menos brusco, logrando que el flujo de aire no tienda a concentrarse únicamente en la parte central del horno, permitiendo atacar de forma directa la zona de recirculación que se presenta en el horno real, como se muestra en las Fig. 9 y 10.

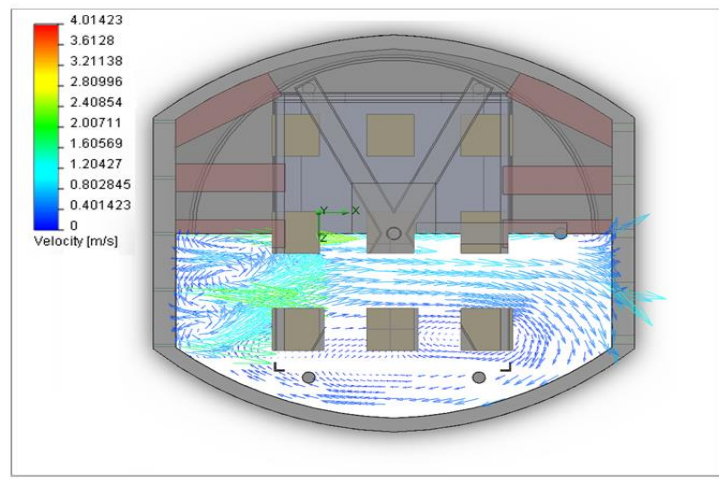

Fig. 9. Campo de velocidad alternativa 2

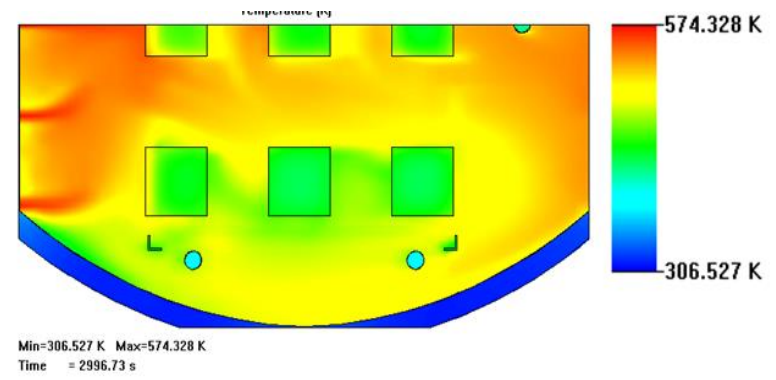

Fig. 10. Campo de temperatura alternativa 2

La Fig. 10 muestra que el patrón de temperatura en esta alternativa tiene cierto grado de similitud con el modelo 1, ya que todavía se verifica una estratificación de la temperatura que se acentúa en la región cercana a la recirculación.

Aunque el tamaño de la zona de recirculación disminuyó un poco aún se observa el influjo de ella en los campos de temperatura, pero hay que resaltar que la uniformidad que se alcanzó en la zona central para el campo de velocidades contribuye notablemente en el equilibrio de las temperaturas que se registran en dicha zona.

Alternativa 3: Horno con mayor número de rejillas de impulsión y mayor área de succión. Esta alternativa se apropia del concepto de la alternativa 2 en lo que respecta a la presencia de curvas laterales, introduce un mayor número de rejillas de impulsión, cuenta con la presencia de deflectores para redistribuir el flujo y enfatiza en el hecho de tener una mayor área de succión. En la Fig. 11, vista superior del horno, se observa más detalladamente las modificaciones que introduce esta alternativa 3 al diseño del horno.

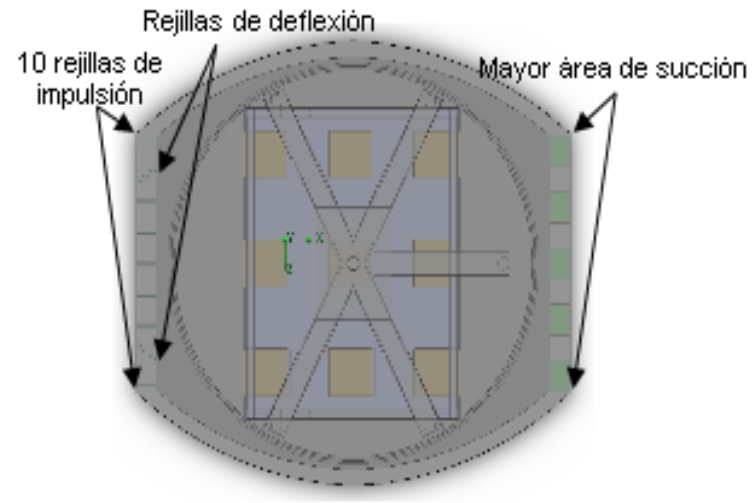

Fig. 11. Vista superior del horno en la alternativa 3 
Las rejillas de impulsión que actúan como deflectores están ubicadas en un ángulo de $33^{\circ}$ con respecto a la horizontal y tienen por objeto redireccionar el flujo hacia las zonas delimitadas por las curvas de las paredes laterales. La mayor área en la succión permite disminuir de manera considerable la restricción a la salida del aire que entregó su energía en forma de calor a los panes, este hecho repercute en forma directa en la zona de recirculación, ya que existirá menos aire que recircule lográndose de esta manera una mayor uniformidad en el flujo de calor al interior del horno, como se muestra en la Fig.12 y Fig.13.

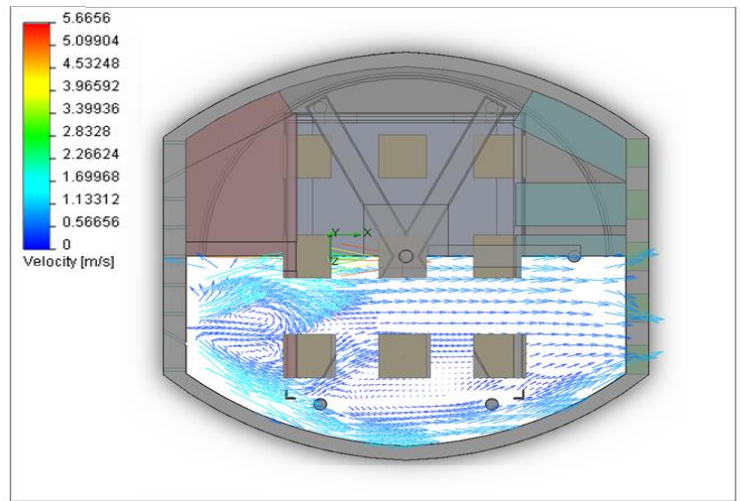

Fig. 12. Campo de velocidad alternativa 3

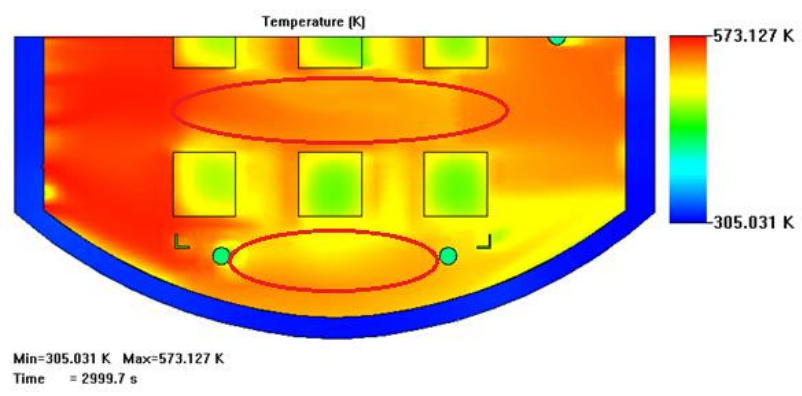

Fig. 13. Campo de temperatura alternativa 3

En esta tercera alternativa desaparece por completo la zona de recirculación aportado significativamente a una uniformidad.

Uniformidad en la temperatura al interior del pan: Un criterio para rediseñar el horno es la uniformidad en la temperatura al interior del pan. Para el estudio se seleccionaron tres panes ubicados estratégicamente al interior del horno, puntos 1,2 y 3 en la fig. 14 .

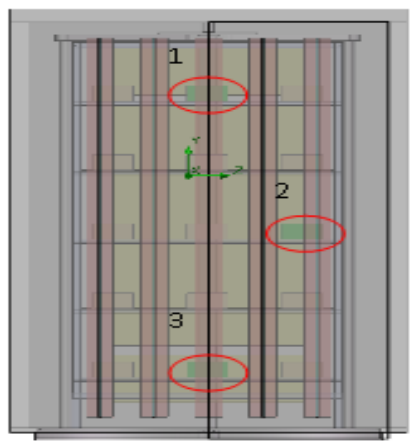

Fig. 14. Ubicación de los panes para el estudio de temperatura al interior del pan (vista lateral)

La temperatura interna del pan desde su cara frontal hasta la posterior en el tiempo final de cocción de 3000 seg, tanto para el horno actual y cada una de las alternativas se muestra en la figura 15.

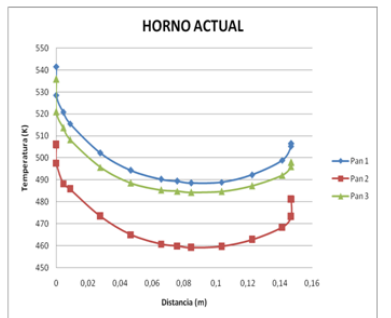

(a)



(c)


Fig. 15. Esquema comparativo de temperatura vs distancia al interior del pan en el tiempo final de la cocción

En la figura 15(a) se observa que el horno actual presenta una gran diferencia de temperatura en los panes como producto final. El pan 2 que se encuentra en la zona de recirculación presenta una temperatura mucho más bajas que los otros dos, los cuales no están en esta zona. La concavidad de las curvas en el horno actual es mucho más pronunciada que en las tres alternativas. Estas alternativas se proponen como una mejora para la uniformidad de la temperatura al interior de los 
distintos panes del lote que entra inicialmente al horno para su cocción. En la Fig. 15 (b) se observa que para la alternativa 1 , la concavidad disminuye un $20 \%$ para cada pan. En la Fig. 15(c) para la alternativa 2 , se observa que el valor aproximado de disminución para cada pan es del 35\% con respecto al horno real. Estos porcentajes de disminución en la concavidad se sacaron tomando los valores de temperatura extremos que se registran en la cara frontal y posterior del pan y relacionándolo con la temperatura en el centro del mismo, la cual representa el valor mínimo en cada curva. En la Fig. 15(d) para la alternativa 3, se observa que las curvas de los tres panes se logran aproximar, la diferencia de temperatura entre estas es de $6^{\circ} \mathrm{K}$ aproximadamente. Estas curvas en la alternativa 3 se ven afectadas de forma directa en la cara frontal con un aumento de su temperatura hasta los $550^{\circ} \mathrm{K}$, razón por la cual se propone que el horno tenga una rotación cuasiestática en el clavijero para que no se vea afectada la uniformidad del flujo [16]. Esa tendencia de las curvas de temperatura en cada pan a aplanarse da a entender que en su interior con cada alternativa logra una uniformidad que no la estaba adquiriendo en el horno real, se da un proceso de mejora en las características de uniformidad del flujo.

Uniformidad de los campos de velocidad: Para comparar el flujo al interior del horno de cada una de las alternativas propuestas, se emplearon ubicación de referencia de manera que brinden información acerca de su comportamiento, esto teniendo en cuenta la criticidad de la uniformidad en las zonas, que en el modelo real se manifiesta en la parte superior e inferior del horno. La Fig. 16 muestra la ubicación 1 y 2 que se tomaron como referencia para el estudio del comportamiento del flujo.

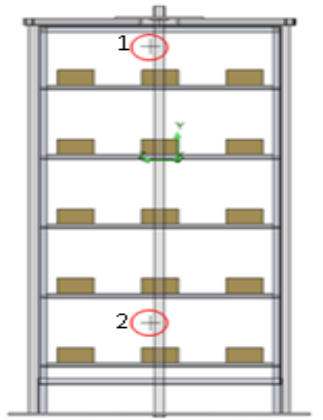

Fig. 16. Ubicación de referencia para el estudio del flujo en el tiempo
En la Fig. 17 y Fig. 18 se detallan la gran oscilación de la velocidad del flujo de aire en el interior del horno.

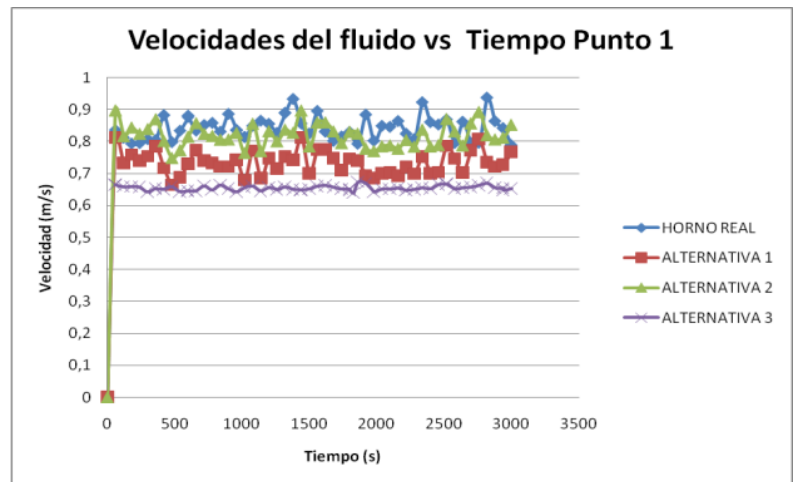

Fig. 17. Variación de la velocidad del fluido en el interior del horno para la ubicación 1



Fig. 18. Variación de la velocidad del fluido en el interior del horno para la ubicación 2

Al presentarse una variación en la velocidad del aire, la transferencia de calor en el pan se ve afectada. Cuando el aire presenta valores de velocidad pequeño, este le entrega calor al pan para llevarlo a un estado de equilibrio, pero producto de los choques se crea turbulencia aumentando su velocidad, lo cual la transferencia solo se da en la superficie del pan logrando que se caliente y no exista una uniformidad del flujo de calor hacia su interior [17].

El horno actual presenta un rango de fluctuación promedio en la parte superior (ubicación 1) de $0.1392 \mathrm{~m} / \mathrm{s}$ y en la parte inferior (ubicación 2) de $0.1820 \mathrm{~m} / \mathrm{s}$ durante el proceso de cocción. Estos valores de oscilación se toman como referencia para evaluar las alternativas propuestas $y$ manifestar un criterio de selección entre ellas (Tabla 3). Se califica el impacto del diseño en la 
uniformidad del flujo por medio de un porcentaje de mejora \%M expresado por la ecuación 6 :

$\% \mathrm{M}=\left[1-\frac{\text { Rango velocidad de cada alternativa }}{\text { Rango velocidad del horno actual }}\right] \mathrm{X} 100$

Tabla 3. Porcentaje mejora de las alternativas de diseño del horno

\begin{tabular}{lllll}
\hline $\begin{array}{c}\text { Rango de } \\
\text { Velocidad } \\
\text { (m/seg) }\end{array}$ & $\begin{array}{c}\text { Horno } \\
\text { Actual }\end{array}$ & Alt. 1 & Alt. 2 & Alt.3 \\
\hline Ubicación 1 & 0.1392 & 0,1349 & 0.1075 & 0.0288 \\
Ubicación 2 & 0.1820 & 0,1434 & 0.1316 & 0.1141 \\
$\begin{array}{l}\text { \% de Mejora } \\
\text { Ubicación 1 } \\
\text { \% de Mejora } \\
\text { Ubicación 2 }\end{array}$ & 3.071 & 22.734 & 79.265 \\
\hline
\end{tabular}

Con los resultados obtenidos se infire que la alternativa 3 presenta mejores caracteristicas de uniformidad del flujo que las del horno actual y demás alternativas. Esta alternativa presentó un porcentaje de mejora de $79.26 \%$ para la ubicación 1 y dé $37.26 \%$ para la ubicación 2.

Selección de la propuesta: La selección de la mejor propuesta se realizó por medio de una matriz de selección, tomando en cuenta los siguientes criterios: costo de la modificación, uniformidad de la temperatura en el pan, uniformidad en el flujo de aire y estética. A estos criterios se le asignaron valores ponderados de 1 a 5 teniendo en cuenta el grado de importancia de cada uno de ellos en el producto. El criterio más relevante es el de la uniformidad de la temperatura en el pan, Como se muestra en la Tabla 4.

Tabla 4. Matriz de selección de la mejor propuesta

\begin{tabular}{cccccc}
\hline Propuesta & Costo & Temp. & Flujo & Estética & Puntaje \\
\hline $\begin{array}{c}\text { Horno } \\
\text { real }\end{array}$ & 2 & 1 & 1 & 3 & 1.4 \\
Alt.1 & 2 & 2 & 2 & 3 & 2.1 \\
Alt.2 & 4 & 4 & 3 & 4 & 3,8 \\
Alt.3 & 3 & 5 & 5 & 4 & 4.5 \\
\hline
\end{tabular}

De la tabla 4 se puede extractar que la mejor propuesta es la alternativa 3, Horno con paredes laterales curvas y con rejillas de impulsión para redireccionamiento del flujo. Aunque el costo de esta alternativa es mayor que la del horno real, esto se compensa por un mejoramiento sustancial de la uniformidad en el flujo y la temperatura con un mejoramiento de la estética del mismo.

\section{CONCLUSIONES}

El estudio realizado del flujo de calor al interior del horno deja claro que el horno actual presenta problemas en la transferencia de calor del aire como fluido caliente hacia los panes como agente receptor. La magnitud de la no uniformidad del flujo de aire se cifra en un $43 \%$ en el horno actual debido a la presencia de una zona de recirculación del flujo que genera desigualdad en los campos de temperatura y velocidad.

Debido a la geometría rectangular que presenta el horno actual, parte del fluido que queda en sus esquinas desarrolla un estado de vorticidad del flujo, lo cual es un problema ya que representa un consumo de energía que no aporta en la transferencia de calor hacia los panes.

Las alternativas propuestas presentaron un incremento en la uniformidad de los patrones de temperatura y velocidad con respecto al horno actual, la alternativa 3 presentó mejoras de hasta un $79.2 \%$, y en el estudio general del flujo de calor y fluido al interior del horno de fabricación de pan se selecciona como la mejor alternativa.

\section{REFERENCIAS BIBLIOGRÁFICAS}

[1] Litovchenko Igor. The Study of the Baking Ovens by Ccomputer Simulation. Acta Universitatis Cibiniensis Series E: Food Technology. Vol. XVII, no.2. 2013. doi: 10.2478/aucft-2013-0018.

[2] Therdthai N, Zhou W, Adamczak T. Two dimensional CFD modeling and simulation of an industrial continuous bread baking oven. J Food Eng. 2003; 60:211-217. doi: 10.1016/S0260-8774(03)00043-8.

[3] Therdthai N, Zhou W, Adamczak T. Threedimensional CFD modeling and simulation of the 
temperature profiles and airflow patterns during a continuous industrial baking process. J Food Eng. 2004; 65:599-608.

doi: 10.1016/j.jfoodeng.2004.02.026.

[4] Zhou W, Therdthai N. Three-dimensional modeling of a continuous industrial baking process. In. Sun DW, editor. Computational fluid dynamics in food processing. Boca Raton: CRC Press; 2007. pp. 287-312. doi: 10.1201/9781420009217.ch11

[5] Wong SY, Zhou W, Hua J. Robustness analysis of CFD model to the uncertainties in its physical properties for a bread baking process. J Food Eng. 2006;77:784-791.

doi: 10.1016/j.jfoodeng.2005.08.019.

[6] Wong SY, Zhou W, Hua J. CFD modeling of an industrial continuous bread-baking process involving U-movement. J Food. Eng. 2007:78:888896. doi: 10.1016/j.jfoodeng.2005.11.033.

[7] Anishaparvin A, Chhanwal N, Indrani D, Raghavarao KSMS, Anandharamakrishnan C. An investigation of bread baking process in a pilotscale electrical heating oven using computational fluid dynamics. J Food Sci. 2010;75:605-E611. doi: 10.1111/j.1750-3841.2010.01846.x.

[8] Mondal A, Datta AK. Two-dimensional CFD modeling and simulation of crustless bread baking process. J Food Eng. 2010;99:166-174. doi: 10.1016/j.jfoodeng.2010.02.015.

[9] Chhanwal N, Tank A, Raghavarao KSMS, Anandharamakrishnan C. Computational fluid dynamics (CFD) modeling for bread baking process-A review. Food Bioprocess Technol. 2012; 5:1157-1172. doi: 10.1007/s11947-012-0804-y.

[10] Sun DW. Computational fluid dynamics in food processing. Boca Raton: CRC Press; 2007. ISBN 9781138568310
[11] Kuriakose R, Anandharamakrishnan C. Computational fluid dynamics (CFD) applications in spray drying of food products. Trends Food Sci Technol. 2010;21:383-398.

doi: 10.1016/j.tifs.2010.04.009.

[12] Gaurav Verma \& Matt Weber. SolidWorks Simulation. 5th Edition. ISBN-13: 978-1988722276. 2018.

[13] Vargas H. Lisandro, Rodriguez P. Alfonso, Castro M. Noel, Pedraza Y. Cristian y Peña C. Jorge. Modelo de la dinámica de fluidos para optimizar el proceso de manufactura de un horno de secado de madera. Revista Ingeniare, Universidad Libre-Barranquilla. Año $9, N^{\circ} 16$, pp 2537. ISSN 1909-2458.doi.org/10.18041/19092458/ingeniare.16.589

[14] Mou B., He B. J., Zhao D. X., Chau K. W. Engineering Application of Computational Fluid Mechanics, Vol 11. 293. 2017.

[15] Liang, C. Papadakis, G. and Luo, X. Effect of tube spacing on the vortex shedding characteristics of laminar flow past an inline tube array, Comput \& Fluids; 38, pp. 950-964, 2009.

doi: 10.1615/JEnhHeatTransf.v17.i3.60

[16] Ateeque, Md., Udayraj, Mishra, R. K., Chandramohan, V. P. y Talukdar, P. Numerical modeling of convective drying of food with spatially dependent transfer coefficient in a turbulent flow field. En: International Journal of Thermal Sciences, 78, pp.145-157. 2014.

doi.org/10.1016/j.ijthermalsci.2013.12.003

[17] Paton, J., Khatir, Z., Thompson H., Kapur, N. y Toropov, $\mathrm{V}$. Thermal energy management in the bread baking industry using a system modelling approach. En: Applied Thermal Engineering, 53(2), pp.340-347. 2013.

doi.org/10.1016/j.applthermaleng.2012.03.036 